ARTIGO ORIGINAL

\title{
Produção e avaliação de briquetes de Schizolobium parahyba var. amazonicum (Huber ex Ducke) Barneby
}

\author{
Production and evaluation of Schizolobium parahyba var. amazonicum \\ (Huber ex Ducke) Barneby briquettes
}

\author{
Vitória Roberta da Silva Ferreira ${ }^{1}$ (1), Pedro Henrique Gonzalez de Cademartori ${ }^{1}$ (D) \\ Edson Alves De Lima² (1D, Fernando Augusto Ferraz ${ }^{1}$ (1), Osmar José Romeiro de Aguiar ${ }^{3}$ (i), \\ Dimas Agostinho Da Silva' ${ }^{1}$ (1) \\ ${ }^{1}$ Universidade Federal do Paraná - UFPR, Curitiba, PR, Brasil \\ Empresa Brasileira de Pesquisa Agropecuária - EMBRAPA FLORESTAS, Colombo, PR, Brasil \\ ${ }^{3}$ Universidade do Estado do Pará - UEPA, Belém, PA, Brasil
}

\begin{abstract}
Como citar: Ferreira, V. R. S., Cademartori, P. H. G., Lima, E. A., Ferraz, F. A., Aguiar, O. J. R., \& Silva, D. A. (2020). Produção e avaliação de briquetes de Schizolobium parahyba var. amazonicum (Huber ex Ducke) Barneby. Scientia Forestalis, 48(128), e3284. https://doi.org/10.18671/scifor.v48n128.12
\end{abstract}

\begin{abstract}
Resumo
A indústria madeireira é caracterizada por produzir grandes quantidades de resíduos através do processo de beneficiamento da madeira. Esses resíduos, se depositados em locais inadequados, podem causar impactos ambientais irreversíveis como a incidência de incêndios. Diante desta problemática, o presente trabalho teve por objetivo investigar o potencial de resíduos do processamento de Schizolobium parayba var. amazonicum (Huber ex Ducke) Barneby para a produção de briquetes. Para tal, foi adotado um esquema fatorial que intercalou os parâmetros através de diferentes pressões $(1000,1300$ e $1600 \mathrm{psi})$, temperaturas $\left(100,130\right.$ e $\left.160^{\circ} \mathrm{C}\right)$ e granulometrias $(40,60$ e 80 mesh), sendo produzidos briquetes a partir de lâminas de alburno e cerne. Inicialmente, determinou-se a transição vítrea da madeira de S. parahyba em um analisador dinâmico-mecânico (DMA). Os briquetes produzidos foram analisados em relação a taxa de retorno em volume, densidade aparente, resistência a compressão e friabilidade. Além disso, determinou-se a composição química imediata e poder calorífico superior, bem como a estabilidade térmica por meio de termogravimetria. A morfologia dos briquetes foi investigada por microscopia eletrônica de varredura. A morfologia dos briquetes evidenciou que maiores temperaturas de processamento resultaram em um material com maior compactação e menor quantidade de espaços vazios e, consequentemente, maior resistência a compressão e menor incidência de finos.Os briquetes produzidos com cerne apresentaram maior poder calorífico superior quando comparados aos briquetes de alburno. A análise química imediata apresentou resultados similares para os briquetes de cerne e alburno, especialmente para teor de voláteis e carbono fixo. A análise de agrupamento evidenciou que a temperatura foi a variável que mais influenciou diretamente as características dos briquetes.
\end{abstract}

Palavras-chave: Biomassa florestal; Biocombustível; Propriedades energéticas; Adensamento; Reaproveitamento de resíduos.

\begin{abstract}
The timber industry is characterized by producing large amounts of waste in the wood processing. Such waste can cause irreversible environmental impacts, if deposited in undesirable places. Thus, the aim of this study was to investigate the potential of processing wood wastes of Schizolobium parayba var. amazonicum (Huber ex Ducke) Barneby to produce briquettes. We adopted a factorial scheme
\end{abstract}

Fonte de financiamento: Coordenação de Aperfeiçoamento de Pessoal de Nivel Superior - CAPES. Número do processo: 88887.498306/2020-00.

Conflito de interesse: Nada a declarar.

Autor correspondente: victoria_roberta19@hotmail.com

Recebido: 12 fevereiro 2019.

Aceito: 4 novembro 2019

Editor: Paulo Henrique Müller Silva.

(c) Este é um artigo publicado em acesso aberto (Open Access) sob a licença Creative Commons Attribution, que permite uso, distribuição e sr reprodução em qualquer meio, sem restrições desde que o trabalho original seja corretamente citado. 
considering different pressures (1000, 1300 and 1600 psi), temperatures $\left(100,130\right.$ and $\left.160{ }^{\circ} \mathrm{C}\right)$ and granulometries (40, 60 and 80 mesh). Briquettes from sapwood and heartwood were produced. First, glass transition temperature of S. parahyba was determined by dynamic mechanical analysis (DMA). The briquettes produced were analyzed in terms of volume contraction index, apparent density, compression strength and friability. Furthermore, immediate chemical analysis and gross calorific value, as well as the thermal stability by thermogravimetry were determined. Morphology of briquettes was investigated by scanning electron microscopy. The morphology of briquettes evidenced that higher processing temperatures resulted in better materials, densification and lower quantity of void spaces and, consequently, higher compression strength and lower incidence of fines. The heartwood briquettes presented higher gross calorific value than sapwood briquettes. Immediate chemical analysis was similar for both sapwood and heartwood briquettes, especially for volatile matter and fixed carbon content. Cluster analysis evidenced the temperature as the parameter of greatest influence in the characteristics of briquettes.

Keywords: Forest biomass; Biofuel; Energetic properties; Densification; Reuse of waste.

\section{INTRODUÇÃO}

A indústria madeireira é caracterizada pela grande quantidade de resíduos de processos de desdobro. Estima-se que do volume total de uma tora seja aproveitado apenas cerca de 40\% a 60\% (Cerqueira et al., 2012). Segundo relatório do Instituto Brasileiro de Árvores (2017), o setor florestal gerou 47,8 milhões de toneladas de resíduos sólidos, sendo que desse total 33,7 milhões, cerca de $70 \%$, foram gerados pelas atividades florestais e 14,11 milhões (aproximadamente 30\%) pelas indústrias. Por conseguinte, é importante ressaltar que o tipo de resíduo gerado no desdobro é caracterizado pela heterogeneidade do material, podendo apresentar cascas e folhas.

Dessa maneira, conhecer as partes da madeira como o cerne e o alburno se faz necessário e de grande importância para determinar a melhor finalidade para a madeira, como a movelaria, produção de papel, dentre outros (Oliveira et al., 2010). A influência de cerne e alburno para o desenvolvimento de carvão vegetal para o setor de energia ainda carece de estudos específicos (Pereira et al., 2013). Da mesma maneira, observa-se situação similar para o setor de produtos energéticos adensados, especialmente os briquetes

Atualmente, diversos setores industriais visam a geração e a utilização de energias renováveis para colaborar com a redução do uso de combustíveis fósseis. Existem alternativas, como a produção de biocombustíveis adensados que visam aproveitar os resíduos gerados, especialmente oriundos do setor florestal e madeireiro. Com isso, esperase contribuir para a racionalização do uso dos recursos florestais, proporcionando uma nova alternativa socioeconômica às empresas, tornando-as ambientalmente adequadas ao gerenciamento de resíduos industriais (Stadler et al., 2009).

Nesse contexto, destaca-se o processo de briquetagem, que consiste na compactação mecânica de resíduos sob elevada pressão e temperatura, resultando em um produto adensado com propriedades energéticas relevantes.

Dessa maneira, a produção de briquetes pode ser uma opção viável para minimizar os problemas sofridos pelo ambiente causados pelo acelerado crescimento das atividades humanas, além de ser uma forma de reutilização dos resíduos utilizados, gerando renda para a indústria, redução do volume de resíduos estacionados no pátio da serraria, poder calorifico superior à lenha, além de diminuir o custo de transporte, auxiliando assim na preservação da natureza e na economia de energia (Gonçalves et al., 2009). Diante do exposto, o objetivo do trabalho foi investigar o potencial de resíduos de lâminas S.parayba (paricá) oriundos do estado do Pará para a produção de briquetes considerando cerne e alburno

\section{MATERIAL E MÉTODOS}

Para a realização deste estudo, foram coletadas lâminas comerciais $(1000 \times 1000 \times 3$ mm) de alburno e cerne de S. parayba no município de Paragominas, sudeste do Estado do Pará(latitude $02^{\circ} 59^{\prime} 45^{\prime \prime}$ Sul e longitude $47^{\circ} 21^{\prime} 10^{\prime \prime}$ Oeste). As lâminas foram transformadas em partículas e posteriormente classificadas de acordo com as granulometrias propostas pelo 
trabalho. A produção dos briquetes foi realizada em uma briquetadeira da marca Lippel, modelo BLB 32. Foram adotadas como condições de processo três temperaturas (100, 130 e $\left.160^{\circ} \mathrm{C}\right)$, três pressões $(1000,1300$ e $1600 \mathrm{PSI})$, além de três granulometrias (40, 60 e 80 mesh), através do esquema fatorial disposto na Tabela 1.

Tabela 1 - TRATAMENTOS UTILIZADOS PARA PRODUÇÃO DOS BRIQUETES

\begin{tabular}{cccc}
\hline Tratamento & Pressão (PSI) & Temperatura $\left.{ }^{\circ} \mathbf{C}\right)$ & $\begin{array}{c}\text { Granulometria } \\
\text { (mesh) }\end{array}$ \\
\hline T1 - P1000T100G60 & $-1(1000)$ & $-1(100)$ & $0(60)$ \\
T2 - P1600T100G60 & $1(1600)$ & $-1(100)$ & $0(60)$ \\
T3 - P1000T160G60 & $-1(1000)$ & $1(160)$ & $0(60)$ \\
T4 - P1600T160G60 & $1(1600)$ & $1(160)$ & $0(60)$ \\
T5 - P1000T130G40 & $-1(1000)$ & $0(130)$ & $-1(40)$ \\
T6 - P1600T130G40 & $1(1600)$ & $0(130)$ & $-1(40)$ \\
T7 - P1000T130G80 & $-1(1000)$ & $0(130)$ & $1(80)$ \\
T8 - P1600T130G80 & $1(1600)$ & $0(130)$ & $1(80)$ \\
T9 - P1000T100G40 & $-1(1000)$ & $-1(100)$ & $-1(40)$ \\
T10 - P1300T160G40 & $0(1300)$ & $1(160)$ & $1(80)$ \\
T11 - P1300T100G80 & $0(1300)$ & $-1(100)$ & $1(80)$ \\
T12 - P1300T160G80 & $0(1300)$ & $1(160)$ & $0(60)$ \\
T13 - P1300T130G60 & $0(1300)$ & $0(130)$ & \\
\hline
\end{tabular}

O tempo de briquetagem foi fixado em 5 minutos, com um período de resfriamento de 10 minutos. Para a produção individual dos briquetes foram utilizadas $20 \mathrm{~g}$ de partículas.

Foi realizada a análise dinâmico-mecânica (DMA) da madeira no equipamento TA DMA Q800. Para tal ensaio, o equipamento foi configurado para operação com frequência de $1 \mathrm{~Hz}$ e taxa de aquecimento de $5^{\circ} \mathrm{Cmin}^{-1}$. Amostras de $S$. parahyba com dimensões de $60 \times 13 \times 20 \mathrm{~mm}$ foram expostas a cargas cíclicas entre 30 e $300^{\circ} \mathrm{C}$.

Para caracterização dos briquetes foi calculado a taxa de retorno em volume através da mensuração de comprimento e diâmetro após a confecção dos briquetes e mensurado novamente após 24 horas. A densidade aparente dos briquetes foi calculada através da norma NBR 14984 (Associação Brasileira de Normas Técnicas, 2003).

Para determinar a resistência a compressão utilizou-se o uma máquina universal de ensaios mecânicos Emic DL30000N. Foram utilizados três briquetes de cada tratamento seguindo as diretrizes da NBR 7222/11 (Associação Brasileira de Normas Técnicas, 2011) adaptada aos briquetes. Enquanto para determinar a durabilidade mecânica ao atrito foi utilizado um corpo-de-prova que foi submetido a um ensaio de 10 minutos, sendo 5 minutos na posição lateral e 5 minutos na face frontal dos briquetes a 50 rotações por minuto (RPM). Posteriormente, os finos gerados foram classificados em uma peneira de 4 mesh.

A microscopia eletrônica de varredura (MEV) foi realizada em um microscópio eletrônico de varredura modelo JEOL JSM 6360- LV. Foram utilizados briquetes confeccionados com as temperaturas de 100 e $160^{\circ} \mathrm{C}$. Foram adquiridas imagens de alta resolução para cada amostra, em diferentes magnitudes de 500, 1000 e 5000x de magnificação.

A Análise termogravimétrica (TGA) foi realizada na madeira in natura como nos briquetes por meio do equipamento STD Q6. Utilizou-se aproximadamente $2 \mathrm{mg}$ de material que foram expostas a uma atmosfera oxidativa de ar sintético para simulação de um processo de combustão. $O$ fluxo de gás foi fixado em $50 \mathrm{~mL} / \mathrm{min}$, a taxa de aquecimento foi de $10^{\circ} \mathrm{C} / \mathrm{min}$ para um intervalo de temperatura de $30-600^{\circ} \mathrm{C}$.

A determinaçãodo Poder Calorífico Superior (PCS) foi realizada através da norma ASTM D5865-13. Para determinação do teor de voláteis utilizou-se a norma E872-82 (ASTM International, 2013a), para calcular o teor de cinzas seguiu as diretrizes da norma D1102-84 
(ASTM International, 2013b) e para calcular o carbono fixo utilizou-se a norma E870-82 (ASTM International, 2013c).

\section{Análise estatística}

Para os ensaios de taxa de retorno em volume, perda de massa e densidade aparente foi realizado a análise de agrupamento no software MATLAB R2018b. Para o ensaio de módulo de ruptura foi empregado a análise de variância no software Statistica 12.

\section{RESULTADOS E DISCUSSÃO}

\section{Análise dinâmico-mecânica}

Através da Figura 1, que explana a análise dinâmico-mecânica (DMA), é possível observar que o ponto de transição vítrea da lignina para a amostra de cerne da madeira de S. parahyba a $9 \%$ de umidade foi de $160^{\circ} \mathrm{C}$, enquanto para amostra de madeira de cerne com $30 \%$ foi de $120^{\circ} \mathrm{C}$. Esse resultado ratifica os dados obtidos por este estudo que mostra que a ponto ótimo da temperatura para a produção dos briquetes de cerne é em torno de $130^{\circ} \mathrm{C}$. No entanto, não foi possível verificar o ponto de transição vítrea para a amostra de alburno. Essa limitação está relacionada a menor resistência mecânica as cargas cíclicas e aumento da temperatura, bem como a difícil visualização do ponto máximo de inflexão no parâmetro tan( $(\delta)$, sendo esta última como possível relação ao menor teor de lignina presente no alburno em comparação ao cerne (Figura 1).

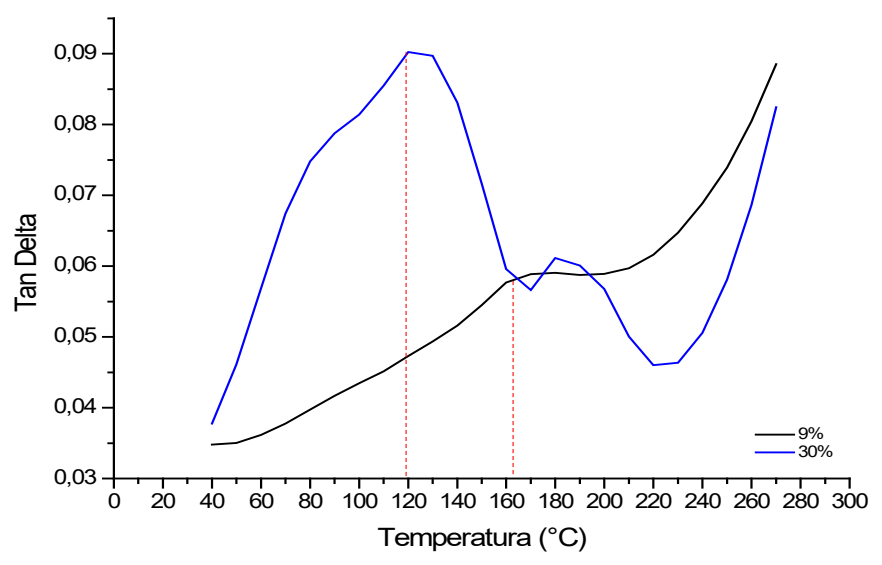

Figura 1 - análise dinâmico-mecânica da madeira de s. parahyba em duas condições de umidade.

É importante destacar que dependendo da umidade da madeira o ponto de transição vítrea pode variar, ocorrendo em temperatura menores, em torno de 60 a $95^{\circ} \mathrm{C}$ (Irvine, 1984; Fengel \& Wegener, 1989; Bhuiyan et al., 2000; Sivonen et al., 2002; Wikberg \& Liisamaunu, 2004; Poncsák et al., 2006). Outro fator que pode contribuir para a transição vítrea da lignina é o seu peso molecular. A temperatura de transição vítrea aumenta com o incremento do peso moleculardos materiais. De acordo com Tolbert et al. (2014), a não-uniformidade das cadeias relacionada ao tamanho das cadeias da estrutura da lignina impedem a caracterização de um peso molecular específico. A estrutura e a composição química da lignina variam em diferentes espécies vegetais, ou em função do método utilizado para extraíla do material lignocelulósico ou processá-la para aplicações posteriores (Ragauskas et al., 2014). Isso corrobora com as diferenças comumente encontradas para o peso molecular da lignina, e consequentemente, na sua temperatura de transição vítrea, a qual pode variar em função, além do peso molecular, de características físicas como a presença de água e o 
histórico térmico do material. Por exemplo: Yoshida et al. (1987) observaram um aumento da temperatura de transição vítrea de $32^{\circ} \mathrm{C}$ para $173{ }^{\circ} \mathrm{C}$ em função do aumento do peso molecular de frações extraídas com solventes orgânicos a partir de lignina Kraft oriunda de licor negro de coníferas.

\section{Análise termogravimétrica}

Através das curvas da madeira in natura e do briquete, é possível observar que até $100^{\circ} \mathrm{C}$ se refere, principalmente a evaporação da água e de outras substâncias com baixa massa molar, como os voláteis, por exemplo. A Figura 2 mostra que a partir de 250 até $350^{\circ} \mathrm{C}$ ocorre uma queda brusca e acentuada de massa. De acordo com Carrier et al. (2011) isso ocorre devido a decomposição dos principais componentes da madeira. Riegel et al. (2008) afirmam que nesse estágio, ocorre intensa cisão de cadeias poliméricas da celulose, acompanhada da decomposição da lignina que apresenta ampla faixa de temperatura, iniciando a degradação em torno de $100{ }^{\circ} \mathrm{C}$, entretanto apresenta taxa reduzida, podendo se estender até $900^{\circ} \mathrm{C}$. A decomposição da celulose o processo dominante dessa etapa (Yang et al., 2007.

A partir de $350^{\circ} \mathrm{C}$ a perda de massa diminui. Issopossivelmente tem relação com a degradação da lignina (Garcia et al., 2016) (Figura 2).
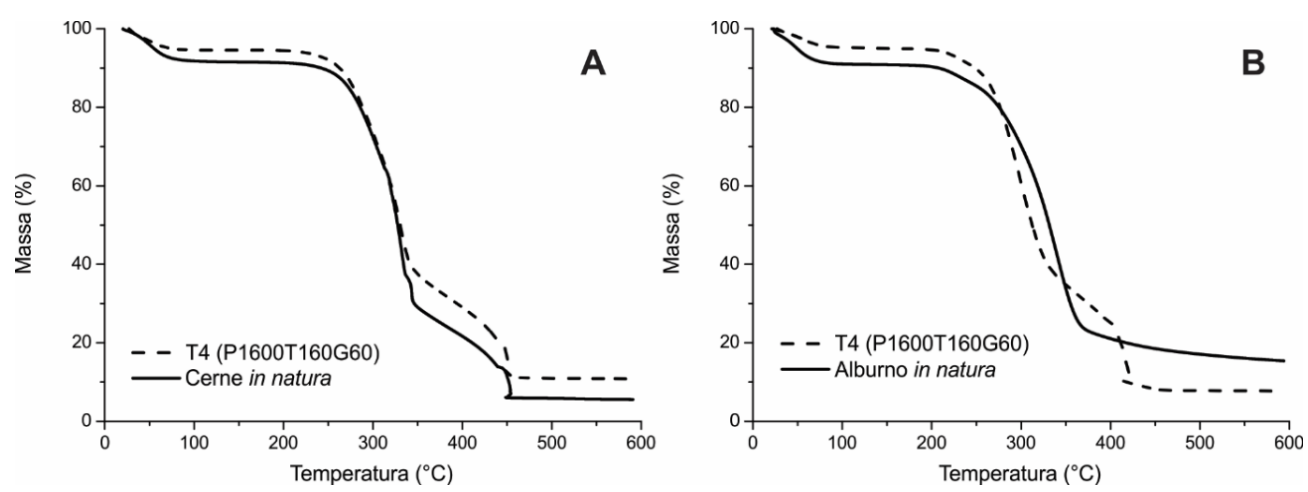

Figura 2 - termogravimetria de madeira in natura e briquetes de cerne alburno de S. parahyba

\section{Caracterização energética}

Na Tabela 2 observa-se que o teor de voláteis e o carbono fixo dos briquetes não mostraram diferença entre os tratamentos, tanto para os briquetes, quanto para madeira in natura. Porém, o mesmo não ocorreu para as cinzas, da qual variou o percentual tanto para os briquetes de alburno e cerne, quanto para madeira.

De acordo com Nogueira \& Lora (2010), quanto menor o teor de voláteis, melhor será para a madeira, pois esta tende a queimar mais lentamente. Dias Júnior et al. (2014), afirmam que altos teores de materiais voláteis são desejados em situações que necessitem de formações de labaredas como lareiras, olarias e fornalhas, sendo dessa forma, recomendados para os referidos usos.

Com relação ao carbono fixo para os briquetes de alburno os valores variaram entre 19,02 a $21,22 \%$ e para o cerne 18,43 a $20,6 \%$. Os valores encontrados para esse trabalho estão de acordo com o encontrado em literatura para diferentes espécies comerciais (Eloy et al., 2015; Nones et al.., 2015; Brand et al., 2013). Tabarés et al. (2000), concluiu que o carbono fixo é o maior contribuinte de fonte energética quando a biomassa é aplicada como combustível, pois aumenta a produção de energia durante a combustão e o rendimento energético do combustível nos fornos.

Para os briquetes de alburno, o percentual de cinzas variou de 0,59 a 0,87\%. Em valores absolutos, é possível observar que no alburno, os tratamentos que geraram maior teor de cinzas, cerca de 0,87\%(T7- P1000T130G80 e T8 - P1600T130G80) e o tratamento que gerou menor teor de cinzas, cerca de 0,59\%(T5 - P1000130G40) apresentou um acréscimo de 32\% no percentual de cinzas. Para o cerne o percentual de cinzas variou 0,53 a 0,83\%.Em valores 
absolutos entre os tratamentos que mais e menos geraram teor de cinzas, houve um acréscimo de $36 \%$ no percentual de cinzas. Estas variações possivelmente estão relacionadas a fatores intrínsecos e extrínsecos à madeira, tais como parte da estrutura vegetal utilizada, tipo de solo e condições climáticas no local de plantio das árvores, e condições de coleta e armazenamento do material, o que faz com que as propriedades das cinzas sejam muito variáveis (Demeyer et al., 2001). Não se utilizou condições de processamento, especialmente temperatura na produção de briquetes passíveis de influenciar o teor de cinzas.

Tabela 2. TEOR DE VOLÁTEIS, CINZAS, CARBONO FIXO E PODER CALORÍFICO SUPERIOR DE CERNE E ALBURNO PARA MADEIRA IN NATURA E PARA OS BRIQUETES DE S. parahyba

\begin{tabular}{|c|c|c|c|c|}
\hline \multicolumn{5}{|c|}{ ALBURNO } \\
\hline Tratamento & Voláteis (\%) & Cinzas (\%) & $\begin{array}{c}\text { Carbono Fixo } \\
(\%)\end{array}$ & P.C.S (kcal.kg-1) \\
\hline Madeira in natura & 78,41 & 0,86 & 20,73 & 4357 \\
\hline T1 - P1000T100G60 & 79,31 & 0,68 & 20,01 & 4000 \\
\hline T2 - P1600T100G60 & 80,16 & 0,72 & 19,12 & 4236 \\
\hline T3 - P1000T160G60 & 78,76 & 0,79 & 20,45 & 4230 \\
\hline T4 - P1600T160G60 & 79,62 & 0,81 & 19,56 & 3960 \\
\hline T5 - P1000T130G40 & 79,59 & 0,59 & 19,82 & 4338 \\
\hline T6 - P1600T130G40 & 78,26 & 0,82 & 20,92 & 4216 \\
\hline T7 - P1000T130G80 & 78,79 & 0,87 & 20,35 & 4209 \\
\hline T8 - P1600T130G80 & 78,28 & 0,87 & 20,85 & 4090 \\
\hline T9 - P1000T100G40 & 80,36 & 0,62 & 19,02 & 4198 \\
\hline T10 - P1300T160G40 & 80,11 & 0,68 & 19,21 & 3846 \\
\hline T11 - P1300T100G80 & 78,16 & 0,84 & 21,00 & 4277 \\
\hline T12 - P1300T160G80 & 79,41 & 0,84 & 19,75 & 4262 \\
\hline T13 - P1300T130G60 & 78,07 & 0,71 & 21,22 & 4284 \\
\hline \multicolumn{5}{|c|}{ CERNE } \\
\hline Tratamento & T. Voláteis (\%) & Cinzas (\%) & C. Fixo (\%) & $\begin{array}{c}\text { P. calorífico } \\
\left(\text { kcalkg }^{-1}\right)\end{array}$ \\
\hline Madeira in natura & 82,5 & 0,35 & 17,5 & 4422 \\
\hline T1 - P1000T100G60 & 79,66 & 0,65 & 19,68 & 4786 \\
\hline T2 - P1600T100G60 & 81,04 & 0,53 & 18,43 & 4745 \\
\hline T3 - P1000T160G60 & 79,01 & 0,60 & 20,40 & 4651 \\
\hline T4 - P1600T160G60 & 80,26 & 0,54 & 19,20 & 4630 \\
\hline T5 - P1000T130G40 & 79,89 & 0,58 & 19,52 & 4642 \\
\hline T6 - P1600T130G40 & 80,58 & 0,65 & 18,76 & 4815 \\
\hline T7 - P1000T130G80 & 79,69 & 0,80 & 19,2 & 4330 \\
\hline T8 - P1600T130G80 & 79,48 & 0,79 & 19,74 & 4766 \\
\hline T9 - P1000T100G40 & 80,59 & 0,53 & 18,88 & 4559 \\
\hline T10 - P1300T160G40 & 80,27 & 0,57 & 19,16 & 4852 \\
\hline T11 - P1300T100G80 & 79,67 & 0,78 & 19,5 & 4667 \\
\hline T12 - P1300T160G80 & 78,31 & 0,83 & 20,6 & 4617 \\
\hline T13 - P1300T130G60 & 79,37 & 0,74 & 19,89 & 4573 \\
\hline
\end{tabular}

Legenda: P: pressão; T: temperatura; G: granulometria; T. voláteis: Teor de voláteis; C.Fixo: carbono fixo; P. calorifico; P.C.S: Poder calorífico Superior.

Outra variável de grande importância para a caracterização energética é o poder

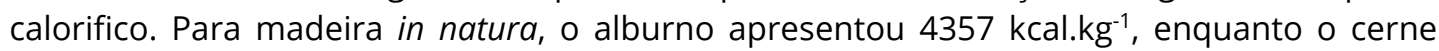


apresentou $4422 \mathrm{kcal} . \mathrm{kg}^{-1}$. Esses valores foram semelhantes ao encontrados para os briquetes produzidos com cerne e alburno.

Os resultados de PCS mostraram que houve diferença entre os briquetes produzidos com cerne e alburno. Para o alburno, o poder calorífico superior variou entre 3846 e 4338 kcal. $\mathrm{kg}^{-1}$, enquanto no cerne variou entre 4330 e $4815 \mathrm{kcal}^{\mathrm{kg}}{ }^{-1}$. Para ambas as partes, a diferença entre o maior e menor poder calorífico foi em torno de $480 \mathrm{kcal}^{\mathrm{kg}} \mathrm{kg}^{-1}$.

É importante observar que os briquetes de alburno apresentaram menor poder calorífico em comparação aos briquetes de cerne. Para efeito comparativo, fazendo a diferença entre o maior poder calorífico dos briquetes de alburno ( $\left.4338 \mathrm{kcal} \mathrm{kg}^{-1}\right)$ e o maior poder calorífico dos briquetes de cerne (4852 $\left.\mathrm{kcal}^{\mathrm{kg}}{ }^{-1}\right)$, observa-se um valor em torno de $514 \mathrm{kcal}^{\mathrm{kg}}{ }^{-1}$, ou seja, $10 \%$ de diferença. Esse resultado já era esperado, pois o cerne possui maior quantidade de extrativos, sendo que estes são formados durante a transição do alburno e cerne resultando em uma coloração mais escura (Oliveira et al., 2005). Outro fator preponderante para o maior poder calorífico do cerne é maior quantidade de lignina comparado ao alburno. Esses fatores são considerados de grande relevância para o aumento do poder calorífico superior (Costa et al., 2017).

\section{Taxa de retorno em volume, densidade aparente e perda de massa}

Através da análise de agrupamento foi possível observar e formar grupos de acordo com as variáveis utilizadas na produção de briquetes. Para os briquetes de alburno, com exceção do tratamento13, e dos tratamentos 3 e 10 formaram um grupo, estes de temperatura de $160{ }^{\circ} \mathrm{C}$. Um segundo grupo foi formado com os tratamentos 9 (P1000T100G40) e 11 (P1300T100G80), com granulometrias e pressões distintas, este grupo apresentava como variável comum a temperatura de $100^{\circ} \mathrm{C}$. O terceiro grande grupo é formado pelos demais tratamentos (Figura 3).

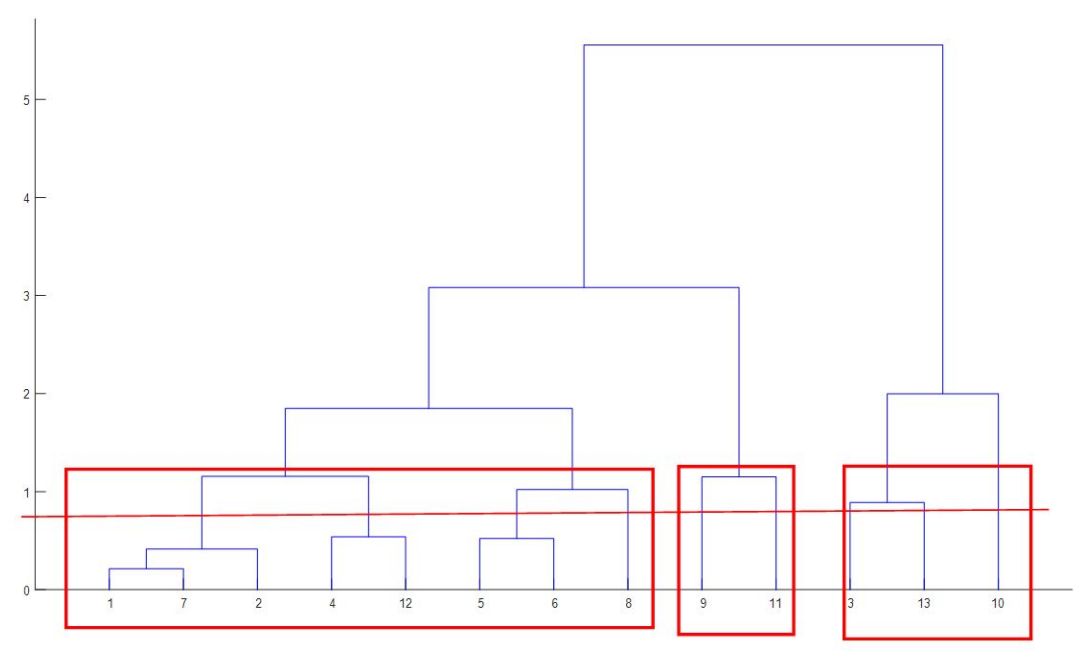

Figura 3 - análise de agrupamento para briquetes de alburno de S. parahyba

O mesmo foi realizado para os briquetes de cerne. Foi possível observar que o primeiro grande grupo é formado pelos tratamentos 3 (P1000T160G60), 4 (P1600T130G40) e 10 (P1300T160G40) que apresentam maior temperatura $\left(160^{\circ} \mathrm{C}\right)$. Um outro grupo foi formado com os tratamentos 8 (P1600T130G80) e 9 (P1000T100G40) com os valores das três variáveis diferentes. E um terceiro grupo foi formado com os tratamentos 1(P1000T100G60) e 2 (P1600T100G60) que possuíam a menor temperatura $\left(100^{\circ} \mathrm{C}\right)$. Os demais tratamentos foram separados em pequenos grupos (Figura 4). 


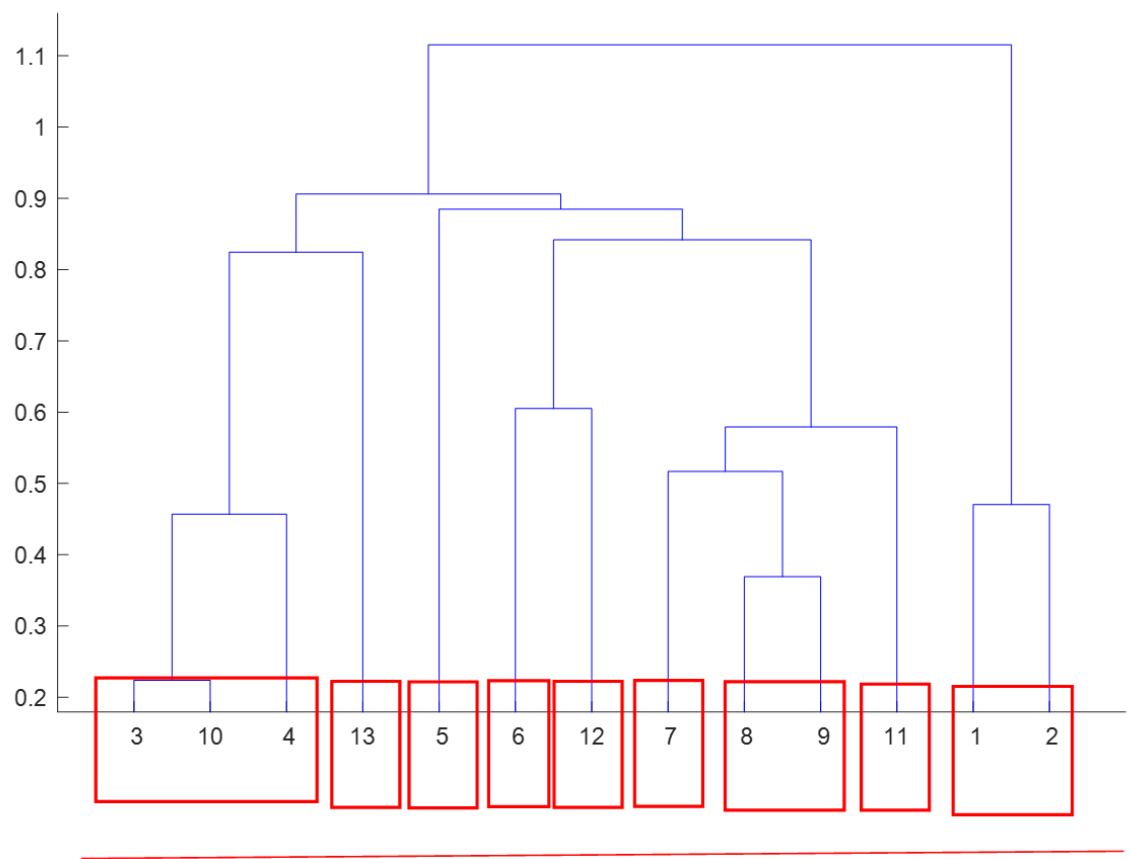

Figura 4 - análise de agrupamento para briquetes de cerne de s. parahyba

\section{Microscopia eletrônica de varredura}

Devido a temperatura ter sido a variável que mais influenciou na densificação dos briquetes, foi realizado a MEV dos briquetes de alburno e cerne com a menor e maior temperatura, ou seja, 100 e $160{ }^{\circ} \mathrm{C}$, respectivamente. Como é possível observar, tanto no cerne, quanto no alburno, com a temperatura de $100^{\circ} \mathrm{C}$ (Imagens A, B, C, D, E, F), as partículas estão menos adensadas, com espaços maiores entre elas, enquanto que na temperatura de $160^{\circ} \mathrm{C}$ (imagens $\mathrm{A}, \mathrm{B}, \mathrm{C}, \mathrm{D}, \mathrm{E}, \mathrm{F}$ ) ocorre uma diminuição desses espaços, deixando as partículas mais adensadas (Figuras 5 e 6 ).
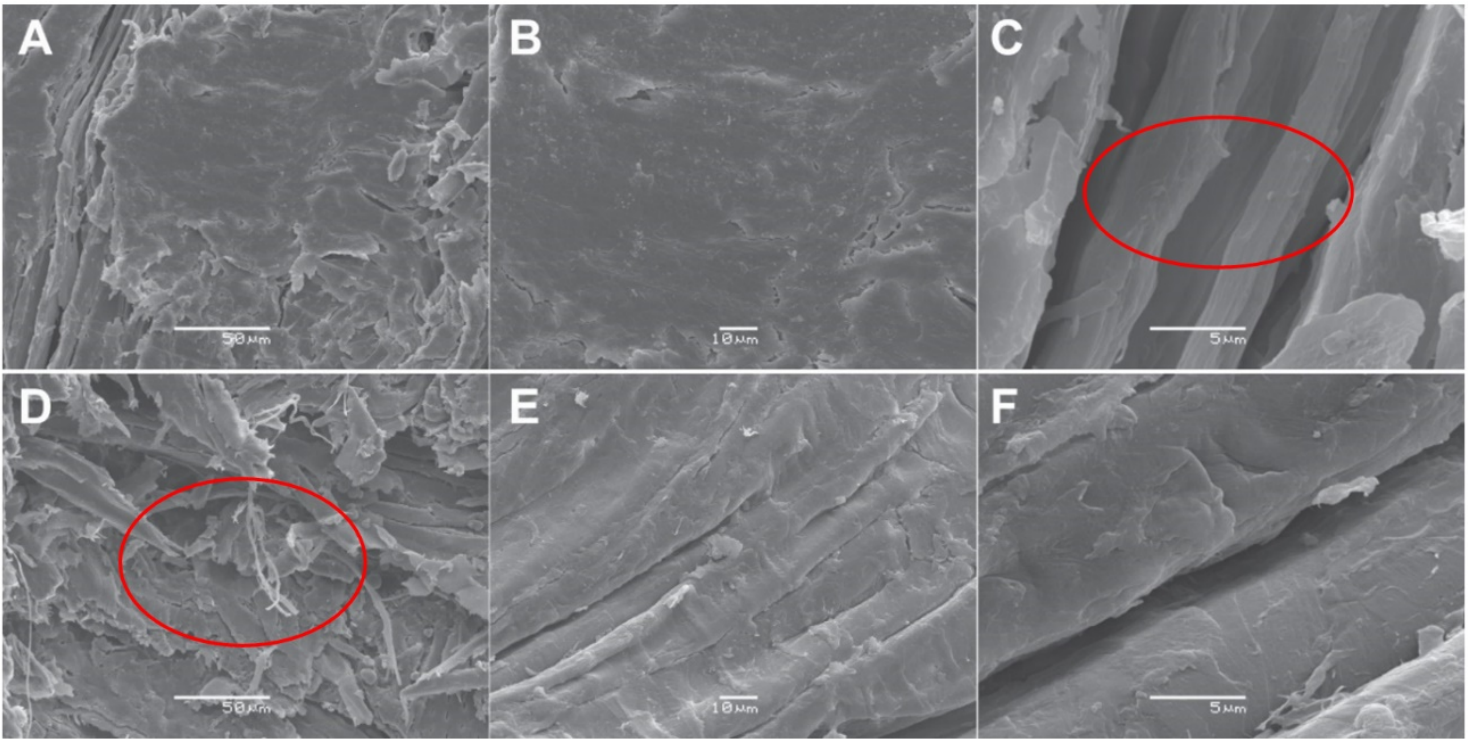

Figura 5 - microscopia eletrônica de varredura dos briquetes dealburno produzidos a $100{ }^{\circ} \mathrm{C}$

NOTA: Briquete produzido nas condições de 60 mesh e 1000 PSI com 500x (A), 1000x (B) e 5000x (C) de ampliação. Briquete produzido nas condições de de 60 mesh e 1600 PSI com 500x (D), 1000x (E) e 5000x

(F) de ampliação. 


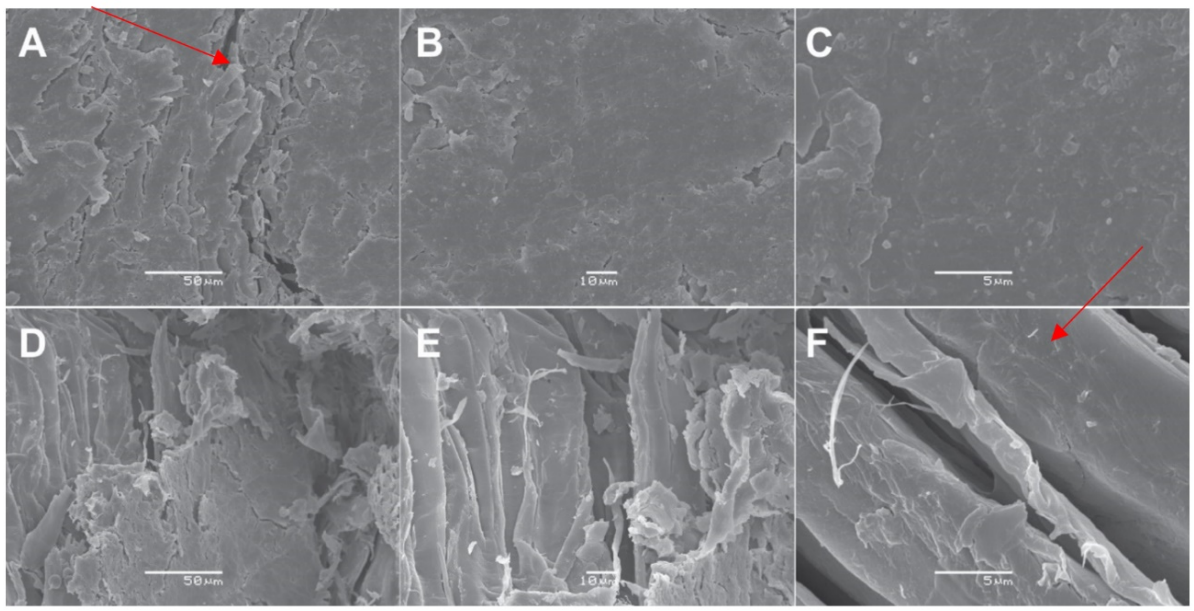

Figura 6 - microscopia eletrônica de varredura dos briquetes de alburno produzidos a $160{ }^{\circ} \mathrm{C}$. FONTE: O autor (2018). NOTA: Briquete produzido nas condições de 60 mesh e 1000 PSI com 500x (A), 1000x (B) e 5000x (C) de ampliação. Briquete produzido nas condições de de 60 mesh e 1600 PSI com 500x (D) 1000x (E) e 5000x (F) de ampliação.

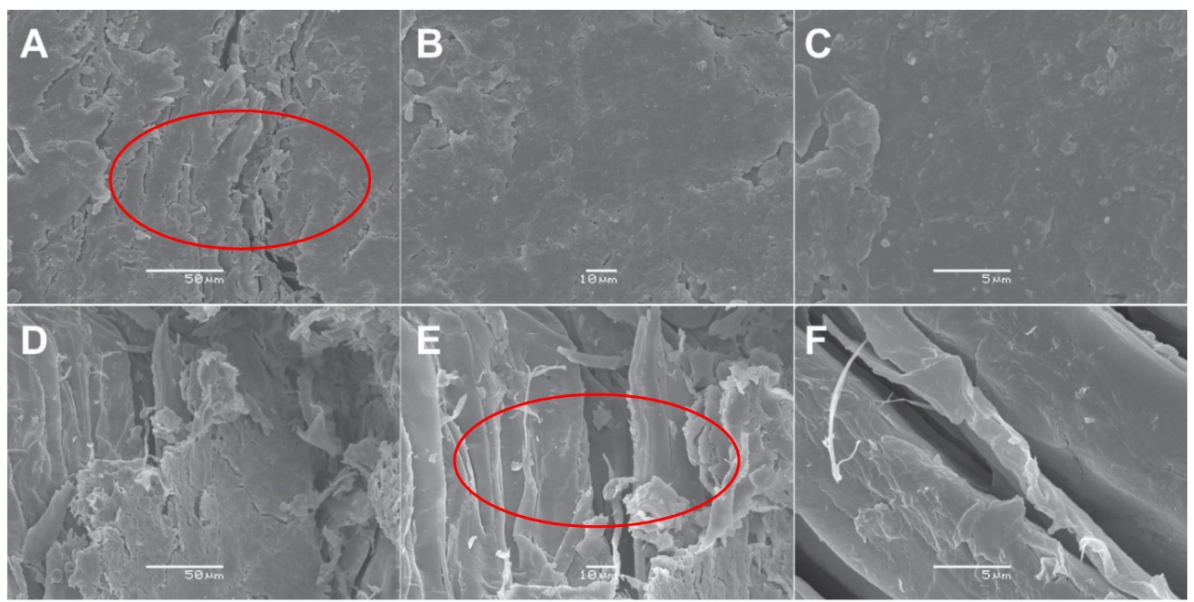

Figura 7 - microscopia eletrônica de varredura dos briquetes de cerne produzidos a $100{ }^{\circ} \mathrm{C}$. FONTE: O autor (2018). NOTA: Briquete produzido nas condições de 60 mesh e 1000 PSI com 500x (A), 1000x (B) e 5000x (C) de ampliação. Briquete produzido nas condições de de 60 mesh e 1600 PSI com 500x (D) 1000x (E) e 5000x (F) de ampliação.

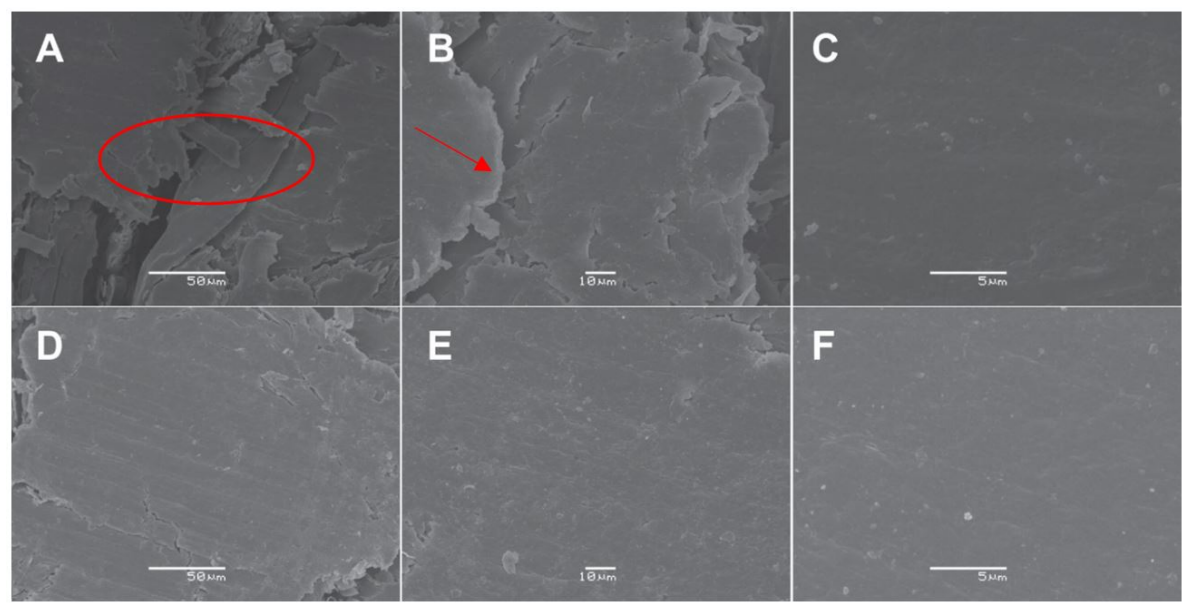

Figura 8 - microscopia eletrônica de varredura dos briquetes de cerne produzidos a $160{ }^{\circ} \mathrm{C}$. FONTE: O autor (2018). NOTA: Briquete produzido nas condições de 60 mesh e 1000 PSI com 500x (A), 1000x (B) e 5000x (C) de ampliação. Briquete produzido nas condições de de 60 mesh e 1600 PSI com 500x (D), 1000x (E) e 5000x (F) de ampliação. 
Nas Figuras 7 e 8 referente ao cerne, mostra que a utilização de condições mais drásticas (temperaturas mais elevadas) reduziu a rugosidade superficial dos briquetes em função do maior adensamento. Além disso estar relacionado com as condições mais drásticas, tem relação parcial com o uso de temperaturas similares ou superiores a transição vítrea da lignina para matéria prima a $12 \%$ de umidade.

Kaliyan \& Morey (2010) avaliaram ligantes naturais e mecanismos de ligação do tipo de ponte sólida em briquetes e pellets feitos de grama e palha de milho. Os autores afirmam que podem ocorrer a formação depontes sólidas entre as partículas devido à cristalização de alguns ingredientes, reação química, endurecimento dos aglutinantes e solidificação dos componentes fundidos. Essas pontes sólidas são formadas principalmente durante o resfriamento e secagem de produtos densificados. Durante o processo de compactação, as fibraspodem se ligar umas sobre as outras, resultando em ligações interligadas.

\section{Resistência a compressão e durabilidade mecânica ao atrito}

Konishi et al. (2017), enfatizaram a importância do teste de resistência a compressão e friabilidade, pois estão diretamente relacionados ao manuseio, transporte e a estocagem do biocombustivel. Observa-se na Tabela 3 que nos briquetes de alburno os tratamentos $3(22,16 \mathrm{MPa}), 4(20,06 \mathrm{MPa})$ e 12 (22,83MPa) apresentaram maior resistência a compressão. É notável observar que para ambos os tratamentos se utilizou uma maior temperatura, porém pressões e granulometrias diferentes.Em termos absolutos, o tratamento com menor resistência (2 - P1600T100G60)) para o tratamento mais resistente (12 - P1300T160G80), houve um acréscimo de 65\% na resistência dos briquetes. Nisto, observa-se que o uso de temperaturas mais baixas interfere na resistência dos briquetes

Para os briquetes de cerne, os tratamentos 3 (P1000T160G60), 4 (P1600T160G60), 8 (P1600T130G80) e 12 (P1300T160G80) apresentaram maior resistência a compressão. Já o tratamento 9 (P1000T100G40) que apresenta menor temperatura mostrou menor resistência a compressão, cerca de $6,74 \mathrm{MPa}$. Novamente, em valores absolutos, mostrou que houve um incremento de $75 \%$ no tratamento menos resistente mecanicamente (9) para o tratamento mais resistente (4).

Dessa maneira, é possível correlacionar uma maior resistência dos briquetes dos tratamentos 3 (P1000T160G60) e 4 (P1600160G60) ao maior adensamento, previamente observados nas imagens de MEV (Figuras 5 e 6), no qual o adensamento tem relação com uma maior adesão entre as partículas e consequentemente uma maior força de ligação interna.

Fernandez et al. (2018) avaliaram as características mecânicas de diferentes tipos de biomassa como resíduos de bagaço de cana e serragem de pinus. Para a resistência a compressão, os autores obtiveram valores entre 0,2 a 0,8 MPa. Vale ressaltar que os autores não utilizaram temperatura para produção de briquetes. Nisto observa-se a importância de uma alta temperatura para confeccionar mais resistente mecanicamente. A resistência a compressão na maioria dos tratamentos deste trabalho mostrou maiores valores quando comparados a literatura.

De acordo com Mariani et al. (2005), o cerne possui maior quantidade de lignina, como foi explanado anteriormente. Além do teor de lignina contribuir com o aumento do poder calorífico superior, a lignina influencia diretamente na resistência dos briquetes. Dessa forma, este fato contribuiu para uma maior resistência a compressão dos briquetes de cerne comparado ao alburno em valores absolutos.

A Tabela 4 mostra o percentual de finos gerados durante o ensaio. É possível notar que os briquetes de cerne geraram menor quantidade de finos que o alburno. Na durabilidade mecânica foi possível verificar que briquetes produzidos com menor temperatura apresentaram maior teor de finos. 
Tabela 3 - MÉDIA, DESVIO PADRÃO E COEFICIENTE DE VARIAÇÃO PARARESISTÊNCIA A COMPRESSÃO DE ALBURNO E CERNE DOS BRIQUETES DE S. parahyba

\begin{tabular}{|c|c|c|}
\hline \multicolumn{3}{|c|}{ ALBURNO (MPa) } \\
\hline Tratamento & Média $\pm \Delta \mathrm{P}(\%)$ & C. V (\%) \\
\hline T1 - P1000T100G60 & $9,01 c \pm 1,20$ & 7,53 \\
\hline T2 - P1600T100G60 & $8,07 c \pm 1,32$ & 6,13 \\
\hline T3 - P1000T160G60 & $22,16 a \pm 1,10$ & 4,26 \\
\hline T4 - P1600T160G60 & $20,06 a \pm 4,69$ & 4,26 \\
\hline T5 - P1000T130G40 & $15,10 b \pm 2,33$ & 6,48 \\
\hline T6 - P1600T130G40 & $13,38 b \pm 0,91$ & 14,58 \\
\hline T7 - P1000T130G80 & $12,71 b \pm 1,81$ & 7,01 \\
\hline T8 - P1600T130G80 & $16,54 b \pm 4,52$ & 3,65 \\
\hline T9 - P1000T100G40 & $12,16 b \pm 0,59$ & 20,53 \\
\hline T10 - P1300T160G40 & $10,33 b \pm 0,70$ & 14,63 \\
\hline T11 - P1300T100G80 & $13,42 b \pm 1,52$ & 8,84 \\
\hline T12 - P1300T160G80 & $22,83 a \pm 3,02$ & 7,56 \\
\hline T13 - P1300T130G60 & $13,42 b \pm 1,49$ & 8,81 \\
\hline \multicolumn{3}{|c|}{ CERNE (Mpa) } \\
\hline Tratamento & Média $\pm \Delta \mathrm{P}(\%)$ & C. V (\%) \\
\hline T1 - P1000T100G60 & $12,00 c \pm 0,32$ & 36,88 \\
\hline T2 - P1600T100G60 & $13,85 c \pm 0,39$ & 34,98 \\
\hline T3 - P1000T160G60 & $23,46 a \pm 1,68$ & 13,12 \\
\hline T4 - P1600T160G60 & $27,26 a \pm 3,37$ & 8,07 \\
\hline T5 - P1000T130G40 & $11,10 c \pm 0,57$ & 19,40 \\
\hline T6 - P1600T130G40 & $13,38 c \pm 0,91$ & 14,58 \\
\hline T7 - P1000T130G80 & $18,55 b \pm 2,08$ & 9,87 \\
\hline T8 - P1600T130G80 & $24,33 a \pm 2,38$ & 10,22 \\
\hline T9 - P1000T100G40 & $6,74 d \pm 0,45$ & 14,72 \\
\hline T10 - P1300T160G40 & $20,74 b \pm 5,45$ & 3,8 \\
\hline T11 - P1300T100G80 & $17,21 b \pm 1,32$ & 13,01 \\
\hline T12 - P1300T160G80 & $24,17 a \pm 1,81$ & 13,32 \\
\hline T13 - P1300T130G60 & $17,48 b \pm 0,95$ & 18,32 \\
\hline
\end{tabular}

LEGENDA: MPa: megapascal; C.V: coeficiente de variação; P: pressão; T: temperatura; G: granulometria; $\Delta P$ : desviopadrão.

Tabela 4 - DURABILIDADE MECÂNICA DE BRIQUETES DE ALBURNO E CERNE DE S. parahyba

\begin{tabular}{ccc}
\hline Tratamento & ALBURNO (\%) & CERNE (\%) \\
\hline T1 - P1000T100G60 & 2,73 & 2,46 \\
T2 - P1600T100G60 & 3,56 & 2,11 \\
T3 - P1000T160G60 & 2,5 & 1,45 \\
T4 - P1600T160G60 & 0,89 & 0,71 \\
T5 - P1000T130G40 & 2,44 & 1,48 \\
T6 - P1600T130G40 & 3,54 & 1,61 \\
T7 - P1000T130G80 & 4,42 & 2,26 \\
T8 - P1600T130G80 & 2,5 & 3,52 \\
T9 - P1000T100G40 & 2,3 & 1,81 \\
T10 - P1300T160G40 & 1,83 & 1,26 \\
T11 - P1300T100G80 & 5,01 & 8,33 \\
T12 - P1300T160G80 & 4,81 & 1,34 \\
T13 - P1300T130G60 & 2,4 & 1,84 \\
\hline
\end{tabular}

LEGENDA: P: pressão; T: temperatura; G: granulometria. 
Todavia, assim como na resistência a compressão, a temperatura se mostrou diretamente proporcional a durabilidade mecânica dos briquetes. Tratamentos com maior temperatura (3 - P1000T160G60, 4 - (P1600T160G60) e 10 - P1300T160G40) geraram um menor percentual de finos, tanto em cerne quanto em alburno. Em termos absolutos, para o alburno, o tratamento que mais gerou finos (12 - P1300T100G80) para o que gerou menos (4 - P1600T160G60) mostrou um valor de acréscimo de $82 \%$ no teor de finos valor. Enquanto no cerne a diferença entre o tratamento que mais gerou finos (11 - P1300T100G80), para o que gerou menos (4 - P1600T160G60) foi de 91\% na geração de finos. Esse fato de tratamentos com maior temperatura gerarem um menor percentual de finos pode ser explicado pelo mesmo motivo da resistência a compressão, da qual trata-se da transição vítrea da lignina, que quando submetida a altas temperaturas, tende a amolecer atuando como um ligante natural, e assim que resfria volta a ter um aspecto enrijecido.

Dessa forma, a lignina deixa os briquetes mais resistentes e menos friáveis. No entanto, os tratamentos 5(P1000T130G40) e 9(P1000T100G40) (que apresentaram maior resistência mecânica, os briquetes de alburno foram mais resistentes do que o cerne), o teste de friabilidade apresentou dados que mostram que os briquetes de alburno foram mais friáveis que os briquetes de cerne.

\section{CONCLUSÃO}

- $\quad$ Através da Microscopia eletrônica de varredura, observou-se que temperaturas mais elevadas formaram briquetes com menor quantidade de espaços vazios;

- $\quad$ As variáveis pressão, temperatura e granulometria não influenciaram nas características energéticas dos briquetes;

- A temperatura foi a variável que mais contribuiu para o adensamento, e resistência a compressão dos briquetes.

\section{AGRADECIMENTOS}

À CAPES pela concessão de bolsa de estudos. À Universidade Federal do Paraná e Embrapa Florestas por ceder a infraestrutura necessária para realização do trabalho.

\section{REFERÊNCIAS}

Associação Brasileira de Normas Técnicas - ABNT. (2003). Madeira - Determinação da densidade aparente dos cavacos. Rio de Janeiro: ABNT.

Associação Brasileira de Normas Técnicas - ABNT. (2011). Concreto e argamassa - Determinação da resistência à tração por compressão diametral de corpos de prova cilíndricos. Rio de Janeiro: ABNT.

ASTM International - ASTM. (2013a). E872-82: Standard Test Method for Volatile Matter in the Analysis of Particulate Wood Fuels. West Conshohocken: ASTM International. Recuperado em 11 de fevereiro de 2019, de https://books.google.com/books?id=JZBNMwEACAAJ\&pgis=1

ASTM International - ASTM. (2013b). D1102-84: Standard Test Method for Ash in Wood. West Conshohocken: ASTM International.

ASTM International - ASTM. (2013c). E870-82: Standard Test Methods for Analysis of Wood Fuels. West Conshohocken: ASTM International.

Bhuiyan, M. R. T., Hirai, N., \& Sobue, N. (2000). Changes of crystallinity in wood cellulose by heat treatment under dried and moist conditions. Wood Science and Technology, 46(6), 431-436. http://dx.doi.org/10.1007/BF00765800.

Brand, M. A., Cunha, A. B., Carvalho, A. F., \& Brehmer, D. R., \& Kuster, L. C. (2013). Análise da qualidade da madeira e do carvão vegetal produzido a partir da espécie Miconia cinnamomifolia (De Candolle) Naudin (Jacatirão-açu) na agricultura familiar, em Biguaçu, Santa Catarina. Scientia Forestalis, 41(99), 401-410.

Carrier, M., Loppinet-Serani, A., Denux, D., Lasnier, J.-M., Ham-Pichavant, F., Cansell, F., \& Aymonier, C. (2011). Thermogravimetric analysis as a new method to determine the lignocellulosic composition of biomass. Biomass and Bioenergy, 35(1), 298-307.

http://dx.doi.org/10.1016/j.biombioe.2010.08.067. 
Cerqueira, P. H. A., Vieira, G. C., Barberena, I. M., Melo, L. C., \& Freitas, L. C. (2012). Análise dos resíduos madeireiros gerados pelas serrarias do município de Eunápolis - BA. Floresta e Ambiente, 19(4), 506510. http://dx.doi.org/10.4322/floram.2012.051.

Costa, A. C. S., Leal, C. S., Santos, L. C., Carvalho, A. M. M. L., Oliveira, A. C., \& Pereira, B. L. C. (2017). Propriedades da madeira de cerne e alburno de Eucalyptuscamaldulensis. Ciência da Madeira, 8(1), 10-20. http://dx.doi.org/10.12953/2177-6830/rcm.v8n1p10-20.

Demeyer, A., Voundi Nkana, J., \& Verloo, M. (2001). Characteristics of wood ash and influence on soil properties and nutrient uptake: an overview. Bioresource Technology, 77(3), 287-295. PMid:11272014. http://dx.doi.org/10.1016/S0960-8524(00)00043-2.

Dias Júnior, A. F., Andrade, A. M., \& Costa Júnior, D. S. (2014). Caracterização de briquetes produzidos com resíduos agroflorestais. Pesquisa Florestal Brasileira, 34(79), 225-234. http://dx.doi.org/10.4336/2014.pfb.34.79.613.

Eloy, E., Silva, D. A., Caron, B. O., \& Souza, V. Q. (2015). Capacidade energética da madeira e da casca de acácia negra em diferentes espaçamentos. Pesquisa Florestal Brasileira, 35(82), 163-167. http://dx.doi.org/10.4336/2015.pfb.35.82.606.

Fengel, D., \& Wegener, G. (1989). Wood chemistry, ultrastructure, reactions (613 p.). New York: Walter de Gruyter Publisher.

Fernandez, B. O., Da Róz, A. L., Gonçalves, B. F., Nakashima, G. T., \& Yamaji, F. M. (2018). Qualidade de briquetes de cana-de-açúcarproduzidos com aglutinante amido de milho. Revista virtual de química, 10(1), 142-154.

Garcia, D. P., Caraschi, J. C., \& Ventorim, G. (2016). Decomposição térmica de pellets de madeira por TGA. Holos., 1, 327-339. http://dx.doi.org/10.15628/holos.2016.3886.

Gonçalves, J. E., Sartori, M. M. P., \& Leão, A. L. (2009). Energia e briquetes produzidos com rejeitos de resíduos sólidos urbanos e madeira de Eucalyptusgrandis. Revista Brasileira de Engenharia Agrícola e Ambiental, 13(5), 657-661. http://dx.doi.org/10.1590/S1415-43662009000500021.

Instituto Brasileiro de Árvores - IBÁ. (2017). Relatório 2017. São Paulo: IBÁ. Recuperado em 11 de fevereiro de 2019, de https://iba.org/images/shared/Biblioteca/IBA_RelatorioAnual2017.pdf

Irvine, G. (1984). The glass transitions of lignin and hemicellulose and their measurement by differential thermal analysis. Journal Tappi, 67(5), 116-121.

Kaliyan, N., \& Morey, R. V. (2010). Natural binders and solid bridge type binding mechanisms in briquettes and pellets made from corn stover and switchgrass. Bioresource Technology, 101(3), 10821090. PMid:19796933. http://dx.doi.org/10.1016/j.biortech.2009.08.064.

Konishi, P. A., Hansted, A. L. S., Nakashima, G. T., Padilla, E. R. D., Da Róz, A. L., Sette Júnior, C. R., \& Yamaji, F. M. (2017). Influência de diferentes condições de armazenamento nas propriedades físicomecânicas de briquetes. Revista Virtual de Química, 9(3). No prelo. Recuperado em 2 de fevereiro de 2019, de http://rvq.sbq.org.br/imagebank/pdf/KonishiNoPrelo.pdf

Mariani, S., Torres, M., Fernandez, A., \& Morales, E. (2005). Effects of Eucalyptus nitens heartwood in kraft pulping. Tappi Journal, 4(2), 8-10.

Nogueira, L. A. H., \& Lora, E. E. S. (2010). Dendroenergia: fundamentos e aplicações (2. ed., 199 p.). Rio de Janeiro: Interciência.

Nones, D. L., Brand, M. A., Da Cunha, A. B., De Carvalho, A. F., \& Weise, S. M. K. (2015). Determinação das propriedades energéticas da madeira e do carvão vegetal produzidos a partir de Eucalyptusbenthamii. Floresta, 45(1), 57. http://dx.doi.org/10.5380/rf.v45i1.30157.

Oliveira, A. C., Carneiro, A. C. O., Vital, B. R., Almeida, W., Pereira, B. L. C., \& Cardoso, M. T. (2010). Parâmetros de qualidade da madeira e do carvão vegetal de Eucalyptuspellita F. Muell. Scientia Forestalis, 38(87), 431-439.

Oliveira, J. T. S., Souza, L. C., Della Lucia, R. M., \& Souza Júnior, W. P. (2005). Influência dos extrativos na resistência ao apodrecimento de seis espécies de madeira. Revista Árvore, 29(5), 819-826. http://dx.doi.org/10.1590/S0100-67622005000500017.

Pereira, B. L. C., Oliveira, A. C., Carvalho, A. M. M. L., Carneiro, A. C. O., Vital, B. R., \& Santos, L. C. (2013). Correlação entre cerne/alburno da madeira de eucalipto, rendimento e propriedades do carvão vegetal. Scientia Forestalis, 41(98), 217-225.

Poncsák, S., Kocaefe, D., Bouazara, M., \& Pichette, A. (2006). Effect of high temperature treatment on the mechanical properties of birch (Betula papyrifera). Wood Science and Technology, 40(8), 647-663. http://dx.doi.org/10.1007/s00226-006-0082-9. 
Ragauskas, A. J., Beckham, G. T., Biddy, M. J., Chandra, R., Chen, F., Davis, M. F., Davison, B. H., Dixon, R. A., Gilna, P., Keller, M., Langan, P., Naskar, A. K., Saddler, J. N., Tschaplinski, T. J., Tuskan, G. A., \& Wyman, C. E. (2014). LigninValorization: ImprovingLigninProcessing in theBiorefinery. Science, 16(6185), 1246843. PMid:24833396. http://dx.doi.org/10.1126/science.1246843.

Riegel, I., Moura, A. B. D., Morisso, F. D. P., \& Mello, F. S. (2008). Análise Termogravimétrica da Pirólise da Acácia-Negra (Acaciamearnsii de Wild.) cultivada no Rio Grande do Sul, Brasil. Revista Árvore, 32(3), 533-543. http://dx.doi.org/10.1590/S0100-67622008000300014.

Sivonen, H., Maunu, L. S., Sundholm, F., Jamsa, S., \& Viitaniemi, P. (2002). Magnetic resonance studies of thermally modified wood. Holzforschung, 56(6), 648-653. http://dx.doi.org/10.1515/HF.2002.098.

Stadler, K., Balbinot, R., Schimer, W. N., \& Vanzetto, S. C. (2009). Análise do processo produtivo e geração de resíduos em uma indústria de painéis compensados. Acta Ambiental Catarinense, 6(1), 45-55.

Tabarés, J. L. M., Ortiz, L., Granada, E., \& Viar, F. P. (2000). Feasibility study of energy use for densificated lignocellulosic material (briquettes). Fuel, 79(10), 1229-1237. http://dx.doi.org/10.1016/S00162361(99)00256-2.

Tolbert, A., Akinosho, H., Khunsupat, R., Naskar, A. K., \& Ragauskas, A. J. (2014). Characterization and analysis of the molecular weight of lignin for biorefining studies. Biofuels, Bioproducts and Biorefining, 8(6), 836-856.

Wikberg, H., \& Liisamaunu, S. (2004). Characterization of thermally modified hard and softwoods by $13 \mathrm{C}$ CPMAS NMR. Carbohydrate Polymers, 58(4), 461-466. http://dx.doi.org/10.1016/j.carbpol.2004.08.008.

Yang, H., Yan, R., Chen, H., Lee, D. H., \& Zheng, C. (2007). Characteristics of hemicellulose, cellulose and lignin pyrolysis. Fuel, 86(12-13), 1781-1788. http://dx.doi.org/10.1016/j.fuel.2006.12.013.

Yoshida, H., Mörck, R., Kringstad, K. P., \& Hatakeyama, H. (1987). Fractionationof Kraft Ligninby Successive Extraction with Organic Solvents. II. Thermal Propertiesof Kraft Lignin Fractions. Holzforschung, 41(3), 171-176. http://dx.doi.org/10.1515/hfsg.1987.41.3.171.

Contribuição dos Autores: VRSF: conceituação, curadoria de dados, análise formal, investigação, metodologia, redação - rascunho original, redação - revisão e edição. PHGC: metodologia, supervisão, validação, redação revisão e edição. EAL: recursos, supervisão. FAF: conceituação, supervisão. OJRA: recursos. DAS: administração do projeto, supervisão, redação - revisão e edição. 